\title{
CHEMOTACTIC TURNING BEHAVIOUR OF TUBULARIA SPERMATOZOA
}

\author{
By R. L. MILLER* AND C. J. BROKAW \\ Division of Biology, California Institute of Technology, \\ Pasadena, California 91 Io9
}

(Received 16 December 1969)

Chemotaxis of spermatozoa to female gonophores has been shown to occur in Campanularia, Tubularia and other hydroids (Miller, I966a,b). Cinemicrographic records of the movements of Campanularia spermatozoa in the vicinity of female gonophores or of glass capillary pipettes containing extracts of female hydranths have shown that chemotactic aggregation of the spermatozoa occurs because the spermatozoa turn and swim toward the source of chemotactic stimulation (Miller, I966a). In the present paper the turning behaviour of hydroid spermatozoa during chemotaxis is examined in greater detail, using multiple-exposure dark-field photomicrography under conditions where the spermatozoa are swimming at an interface between sea water and a glass surface. Under these conditions the changes in bending behaviour of the sperm flagellum can be photographed, in addition to the changes in the path of the spermatozoon. Although this report deals exclusively with spermatozoa of Tubularia, similar results have been obtained from less extensive attempts to photograph the turning behaviour of Campanularia spermatozoa.

\section{MATERIALS AND METHODS}

Spermatozoa were obtained by allowing a few large male hydranths of Tubularia crocea to stand for $10-30 \mathrm{~min}$. in a small volume of filtered sea water containing $0.5 \mathrm{~mm}$-EDTA and $\mathrm{r} \%$ polyvinylpyrrolidinone, with the $\mathrm{pH}$ adjusted to $8 \cdot \mathrm{I}$. Female hydranths were extracted in $95 \%$ ethanol for $\mathrm{i} 2 \mathrm{hr}$. or more. The filtered or decanted supernatant was evaporated to dryness, and the residue was then dissolved in a small amount of $0.5 \mathrm{M}-\mathrm{NaCl}$. These preparations remain active for several weeks. Glass capillary micropipettes, drawn by hand to tip diameters of 50-100 $\mu$, were filled with agar gel dissolved in $0.5 \mathrm{M}-\mathrm{NaCl}$. After the agar had solidified, these micropipettes were allowed to stand with their tips in the female hydranth extract for several days in the refrigerator. They were removed and used as needed, and then returned to storage with their tips in the extract. A micropipette held in a micromanipulator was aligned so that its tip was held just above the surface of a microscope slide within the field of the microscope, and covered with a cover glass supported by fragments of broken coverglass. The resulting chamber was then filled by adding sperm suspension at the side of the coverglass. The slide was moved occasionally to bring a fresh region of sperm suspension into the vicinity of the tip of the micropipette. All observations were made in at room maintained at $16^{\circ} \mathrm{C}$.

\footnotetext{
- Present address: Department of Biology, Temple University, Philadelphia, Pennsylvania 19122.
} 
Photomicrographs were taken on Tri-X film in a Robot $35 \mathrm{~mm}$. camera, using a Zeiss low-power dark-field condenser, and a Chadwick-Helmuth model $135 \mathrm{~N}$ microscope strobe illuminator. The illuminator was allowed to flash continuously at the desired frequency, and the exposure was controlled by opening and closing the camera shutter manually while observing the spermatozoa. Low-power micrographs were made with a $10 \times, 0.32 \mathrm{NA}$ planapochromat objective, with a final magnification of $40 \times$ on film. Higher-resolution micrographs were made with a $40 \times, 0.6 \mathrm{NA}$ oil immersion apochromat objective, with a final magnification of $160 \times$ on film. A flash frequency of $9 \mathrm{~Hz}$, giving about one exposure for every four beats of the sperm flagellum, was used for the low-magnification micrographs. Flash frequencies of 16 , $\mathrm{I}$, or $20 \mathrm{~Hz}$, giving about one exposure for every two beats of the sperm flagellum, were used for the higher-magnification micrographs. Visual observations were also made, at a variety of flash frequencies. In contrast to some species of spermatozoa which we have studied the movement of Tubularia spermatozoa appeared to be completely unaffected by the high-intensity light flashes used for photomicrography.

\section{OBSERVATIONS}

As noted previously with Campanularia spermatozoa (Miller, 1966a) the movements of Tubularia spermatozoa often are not fully activated until after the spermatozoa have been exposed to female gonophores or extracts. When fully activated the sperm flagellum beats at $34-36 \mathrm{~Hz}$, and its pattern of bending waves, shown in several of the photographs in this paper (e.g. Pl. 2, fig. 3), is similar to those produced by other spermatozoa, such as sea-urchin spermatozoa, which have been studied with similar photographic techniques (Brokaw, 1965). The only difference is that the Tubularia spermatozoa used in these experiments were much more frequently found to be swimming in nearly straight paths than the spermatozoa of the species of sea urchins we have examined.

When the spermatozoa are not fully activated the sperm flagellum beats at somewhat lower frequencies $(25-30 \mathrm{~Hz})$ and generates an abnormal wave pattern, such as the one shown in Pl. 2, fig. 6. With these spermatozoa there is usually an obvious increase in swimming velocity when a spermatozoon turns and swims towards a source of chemotactic stimulus, as noted previously with Campanularia spermatozoa (Miller, I $966 a$ ). In the present study we have paid little attention to the activation of motility by hydranth extracts, and have attempted to photograph only the turning behaviour of fully activated spermatozoa. In this state there appears to be little further increase in swimming velocity as the spermatozoon approaches the tip of a micropipette containing female hydranth extract.

The flagellum of a Tubularia spermatozoon, like those of many other marine invertebrate spermatozoa (Gray, I955; Brokaw, 1965), generates nearly planar bending waves. These spermatozoa become trapped at surfaces, over which they swim, without rotating, with the plane of beat of the flagellum parallel to the surface. Because of this behaviour it is possible to observe a particular spermatozoon over an extended period while it swims next to the surface of the microscope slide or coverglass. Under these conditions its orientation is well-defined, and information about both the path of movement of the spermatozoon and the bending behaviour of its flagellum can be recorded in photographs. 


\section{Sperm paths during chemotaxis}

The low-magnification photographs (Pl. $\mathrm{x}$ ) record positions of the sperm head at intervals of $\frac{1}{8}$ sec., and usually show the sperm flagellum clearly enough to define the direction of the path of the sperm head. Aggregations of spermatozoa swimming actively around the tip of a micropipette are visible in some of these photographs (Pl. I, figs. I, 6 and 9).

When spermatozoa swim into the vicinity of a micropipette from which female hydranth extract is diffusing their paths show two distinct types of turns. One type of turn, which will be referred to as an outward turn, starts as a turn away from the tip, but the spermatozoon continues to turn through more than $180^{\circ}$ and usually ends up with a path which is more closely oriented towards the tip. The precision of orientation by outward turning is relatively low, and frequently a series of such turns is required before the path of the spermatozoon carries it to the tip of the micropipette. Examples of outward turns are shown in Pl. I, figs. I- 3 , and others.

The second type of turn, which will be referred to as an inward turn, is initially in the direction which will cause the path of the spermatozoon to point toward the tip of the micropipette. In some cases, such as Pl. I, figs. 4 and 5, turning continues at a low rate so as to continually adjust the path of the spermatozoon and bring it toward the tip. In other cases, such as those shown in Pl. I, figs. 6 and 7, the inward turn is abrupt and may not produce perfect orientation, so that another turn or turns is subsequently required before the spermatozoon reaches the tip. However, the general impression from observations and from the photographs is that inward turning produces more accurate orientation toward the tip than does outward turning. Occasionally, a single spermatozoon has been observed to make both types of turns, as required, as its path approaches the tip. Pl. $x$, fig. 8 may illustrate such a case.

It is usually possible to predict whether a spermatozoon will respond with an inward or an outward turn, because its direction of turning during chemotactic response is usually in the same direction as the steady, lower-level turning which causes most spermatozoa to swim normally in arcs of large radius. Some exceptions to this rule have been observed; two of these are shown in Pl. I, fig. 9. On the other hand, when a succession of chemotactic turns by a particular spermatozoon has been observed, these turns have always been in the same direction relative to the body of the spermatozoon.

Outward turns appear to be far more common than inward turns. This observation may be expected, since an inward turn is most likely to occur in a spermatozoon which is swimming in an arc which curves towards the tip of a micropipette. Such a path is likely to have been closer to the tip at some earlier time, causing the spermatozoon to be 'trapped' near the tip by its chemotactic response. When chemotaxis is observed in a deep drop of sperm suspension inward turns appear to be relatively more frequent, perhaps because 'inward facing' spermatozoa are less likely to have previously passed close to the micropipette.

After a spermatozoon has reached the tip of the pipette it swims actively around in a small region, with very frequent, sharp turns. After several minutes the flagella of these spermatozoa, which have been exposed to a presumably high concentration of chemotactant, often display a different form of bending pattern, shown in Pl. 2, fig. 7 . 
Both the wavelength and radius of curvature of the waves are reduced, and these spermatozoa also beat at somewhat lower than normal frequencies $\left(25^{-}-30 \mathrm{~Hz}\right)$.

\section{Behaviour of the sperm flagellum during turning}

The higher-magnification photographs in Pl. 2, figs. I-5 show the sperm flagellum during chemotactic turns. During a turn the sperm flagellum continues to beat but produces an asymmetrical pattern of bending, which is probably responsible for the turning of the spermatozoa. There are only small changes in frequency of beat during turning. No change in frequency of beat can be detected from the information available for the relatively short turn shown in Pl. 2, fig. 2. In Pl. 2, fig. 1, the spermatozoon appears to decrease its frequency of beat from about $34 \mathrm{~Hz}$ before turning to about $32 \mathrm{~Hz}$ during turning, and to increase its frequency again after completing the turn. The turn shown in Pl. 2, fig. 4 shows a slight decrease in frequency of beat, from approximately $34 \mathrm{~Hz}$ before turning to approximately $32 \mathrm{~Hz}$ during the turn. The spermatozoon in Pl. 2, fig. 5 was also beating at approximately $32 \mathrm{~Hz}$ during the turn, and increased its frequency to approximately $33 \mathrm{~Hz}$ after recovery from turning. These small changes in frequency of beat could be the result of altered mechanical feedback to the frequency-determining oscillator in the base of the flagellum as a consequence of alterations in the pattern of flagellar bending. They do not necessarily indicate a direct effect of chemotactic stimulation on the frequency generator.

The multiple-exposure technique used for these photographs, with approximately one photographic flash per two flagellar beat cycles, gives an incomplete record of the behaviour of the flagellum during turning. It is difficult to reconstruct precisely the complete behaviour by using photographs of a number of turns, since each turn is somewhat different. However, it appears to be possible to obtain a consistent interpretation of the behaviour of the flagellum. The two turns shown in Pl. 2, figs. I and 2 are particularly instructive, since these turns are fairly similar but the photographs are taken at different frequencies. The information in these photographs is perhaps more easily interpreted by the dissected diagrams in Text-figs. I and 2, in which successive images of the spermatozoon have been traced and separated.

The spermatozoon shown in Pl. 2, fig. I and Text-fig. I was beating at approximately twice the photographic flash frequency during the turn, so the photographic sequence shows the development of asymmetrical beating and the return to normal beating throughout the turn at a particular phase of the beat cycle. This photograph indicates that an asymmetrical pattern develops and is maintained for at least four complete beat cycles (positions 7,8 and 9 in Text-fig. 1 ).

The spermatozoon in Pl. 2, fig. 2 and Text-fig. 2 was beating at about 33 beats/sec. and was photographed with flashes at $20 \mathrm{~Hz}$, so that a sequence of patterns at different phases of the beat cycle is shown during the turn. The asymmetrical bending pattern involves an increase in angle of bend, from about $\mathrm{r} .8$ radians up to about 2.4 radians, for the bends on one side of the flagellum (the bends having the same sense of curvature as the turn of the spermatozoon). This increased angle of bend results from a decreased radius of curvature by almost a factor of 2 ; it is accompanied by a slight decrease in the length of the region of bending. The bends produced by the flagellum in the opposite direction are reduced by about $50 \%$ near the base, and down to nearly $o$ as these bends propagate toward the distal end of the flagellum. This decrease 
appears to involve both a decrease in the length of the region of bending and a large increase in the radius of curvature of the bend.

The results of these changes are (i) a gross curvature of the entire spermatozoon, so that the axes of symmetry of bending of the proximal and distal parts of the flagellum are pointing in quite different directions; (ii) a slight decrease in wavelength; and (iii) an appearance of restriction of bending to the proximal end of the flagellum.
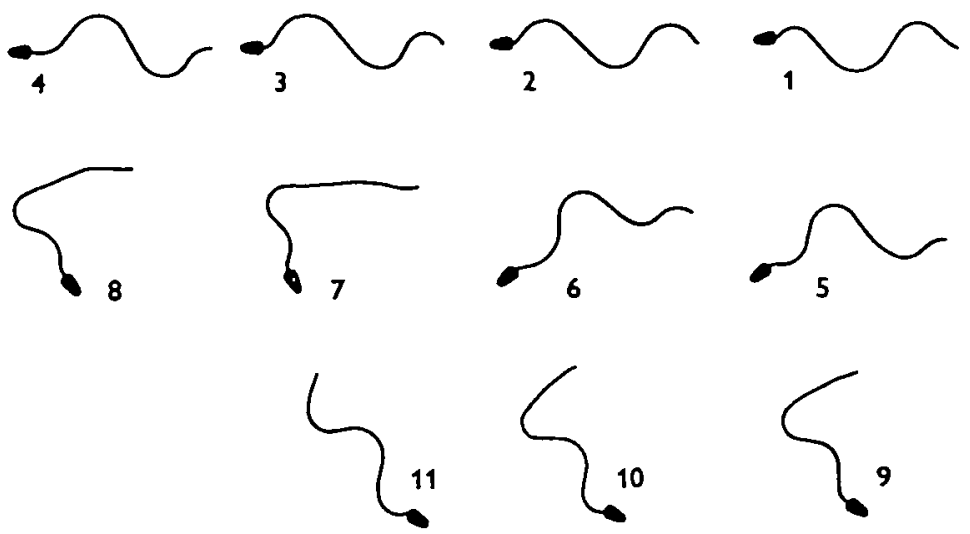

Text-fig. 1. Sperm-tail patterns traced from the photograph shown in Pl. 2, fig. I.
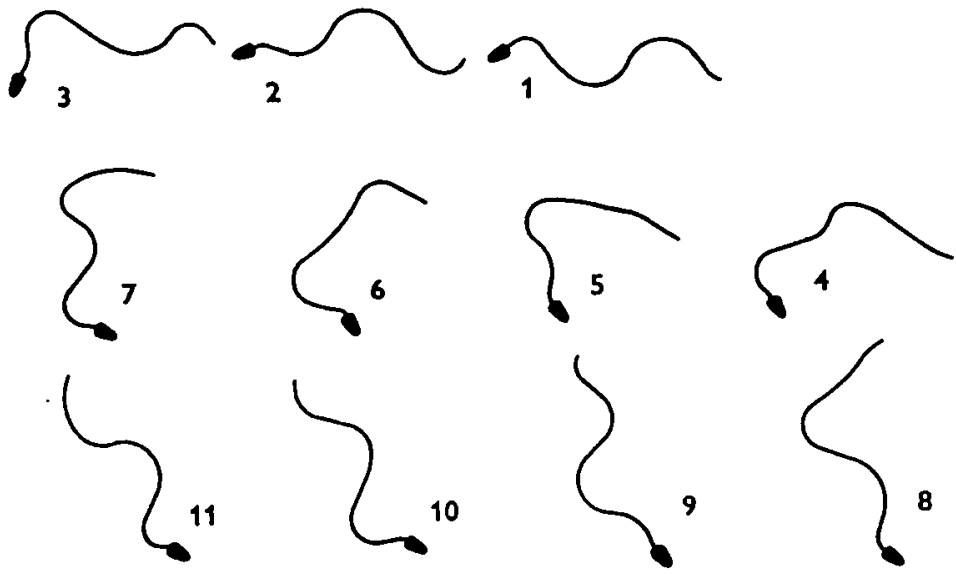

Text-fig. 2. Sperm-tail patterns traced from the photograph shown in Pl. 2, fig. 2.

Although we are interpreting the gross curvature as a consequence of altered degrees of bending in the bent regions only, resolution of the straight regions between bends is not good enough to completely exclude the possibility that the gross curvature of the flagellum is produced by an independent bending mechanism, on which a nearly symmetrical pattern of propagated bends is superimposed.

Recovery from the turn appears to involve a gradual re-involvement of the distal end of the flagellum in propagated bending in both directions, so that symmetrical bending reappears in the distal portion of the flagellum. The entire sequence requires approximately $13^{-15}$ flagellar beat cycles in these two cases. Although other turns 
sometimes involve more or less extreme degrees of asymmetry in beat pattern, the major determinant of the total angle of turn in the path of a spermatozoon appears to be the duration of turning.

\section{DISCUSSION}

Our photographs suggest that in Tubularia spermatozoa chemotaxis is the result of alterations in the symmetry of flagellar beat patterns in response to a chemical stimulus. The magnitude of the response is variable and appears, to some extent, to be matched to the intensity of the stimulus that we might expect the spermatozoon to have received. However, the direction of response does not appear to be determined by directional information contained in the stimulus. Each spermatozoon appears to be able to make turns in only one direction, relative to its own body.

The asymmetry which develops in the beating of the flagella of Tubularia spermatozoa during chemotactic turning is similar in character to the asymmetry found occasionally in the movements of unstimulated spermatozoa. Gray (1955) described a qualitatively similar asymmetry of the bending waves in his original paper on the movement of the sea-urchin sperm flagellum. In sea-urchin spermatozoa, but not in Tubularia spermatozoa, consistently asymmetrical beating can be induced by increasing the potassium ion concentration of the sea water in which they are swimming, and similar asymmetrical beating patterns are also characteristic of the movement of ATP-reactivated, glycerinated, sea-urchin spermatozoa (Brokaw, 1966).

The morphology of flagellar movement during turning in Tubularia spermatozoa appears to be distinctly different from that found during turning of the spores of Blastocladiella (Miles \& Holwill, 1969) or during the chemotactic turning of Allomyces gametes (M. E. J. Holwill, personal communication). In these two cases there appears to be a complete cessation of normal beating; a single asymmetrical bend appears at the base of the flagellum and then the flagellum resumes beating and propels the cell in a new direction. Tubularia spermatozoa, on the other hand, continue to beat asymmetrically for 10 to 20 beats during a turn. In both types of turning, however, the direction of turning is probably fixed with reference to the cell.

In other cases, such as the biflagellate alga, Polytoma (Brokaw, 1963), or ciliated protozoa (Naitoh, 1966), response to stimuli which cause a change in swimming direction involves a transient change in direction of the flagella or cilia, on which a more or less normal pattern of beating is superimposed. In these cases, and possibly in Blastocladiella, there appears to be a 'contractile' mechanism, independent of normal flagellar beating, which controls flagellar orientation and is responsible for turning movements (Naitoh, 1969 ). We have been unable to decide from our photographs whether turning in Tubularia spermatozoa also involves an independent bending mechanism, or whether it involves an alteration in the parameters of operation of the normal mechanism for generating flagellar bending waves.

Many of the turns of Tubularia spermatozoa, particularly those which are outward turns, appear to start at a time when the normal movement of the spermatozoon starts to carry it away from the tip of the micropipette, so that the stimulus for turning could be a decrease in the concentration of chemotactant. Since an outward turn will initially amplify this stimulus, the outward turn becomes very sharp, and continues until the spermatozoon is experiencing an increasing concentration of chemotactant. 
There are other cases, particularly with inward turns, where the response occurs at a point where the concentration of chemotactant should be increasing along the sperm path, but the sperm turns so that it will experience a greater rate of increase of concentration. If sensitivity to decreasing concentration is restricted to a small part of the spermatozoon, the oscillation of this chemoreceptor back and forth across the path of the spermatozoon with each beat of the sperm flagellum might provide a signal of decreasing concentration even though the average path of the spermatozoon was toward increasing concentrations. If the response mechanism can detect these very rapid changes in concentration, turning might be stimulated under such conditions, leading to an improvement in orientation. However, we have no direct evidence to support such a sampling mechanism.

Our observations suggest that the chemotactic behaviour of Tubularia spermatozoa swimming against a surface might be completely explained if the spermatozoa have a mechanism for producing turns in one direction, and if this turning mechanism is activated when the spermatozoon senses a decreasing concentration of chemotactant. When spermatozoa are swimming in a deeper drop of suspension, away from interfaces, they will tend to swim in three-dimensional helical paths, because of their tendency to rotate as they swim. Our impression is that the chemotactic behaviour observed under these conditions is not very different and not significantly more precise than that observed when the spermatozoa are swimming at an interface, and that the mechanism suggested above might be capable of explaining their behaviour.

Although not strictly comparable because of differences in the size of the source relative to the size of the cell, the precision of chemotactic orientation in Tubularia spermatozoa appears to be significantly less than in the chemotaxis of bracken spermatozoids, as illustrated by Brokaw ( $\left.195^{8} a, b\right)$. Bracken spermatozoids do not appear to be restricted to a single direction of turning (Brokaw, 1958b). These differences may simply reflect the fact that bracken spermatozoids have a large number of flagella, which might independently respond by asymmetrical beating, rather than a basic difference in mechanism.

\section{SUM MARY}

I. The movements of Tubularia spermatozoa in the vicinity of micropipettes filled with extracts of female hydranths, which chemotactically attract the spermatozoa, have been recorded by multiple-flash photomicrography.

2. When a spermatozoon turns in response to a chemotactic stimulus, the flagellum continues to beat, with a highly asymmetrical pattern of bending, during the turn.

3. The magnitude of the turn, particularly the duration of the period of asymmetrical beating, is variable, but each spermatozoon is only able to make turns in one direction, relative to its own body.

4. Most of the behaviour of these spermatozoa may be explained if the turning mechanism is activated when the spermatozoon detects a decreasing concentration of the chemotactant.

This work has been supported in part by a grant from the United States Public Health Service (GM-146r3). It is a pleasure to acknowledge the able technicalassistance of Bruce Benedict during many parts of this project. 


\section{REFERENCES}

Brokaw, C. J. (1958a). Chemotaxis of bracken spermatozoids. Implications of electrochemical orientation. F. exp. Biol. 35, 197-212.

Brokaw, C. J. (1958b). Chemotaxis of bracken spermatozoids. Ph.D. Thesis, Cambridge University. Brokaw, C. J. (1963). Movement of the flagella of Polytoma uvella. J. exp. Biol. 40, 149-56.

Brokaw, C. J. (1965). Non-sinusoidal bending waves of sperm flagella. F. exp. Biol. 43, I 55-69.

Brokaw, C. J. (1966). Effects of increased viscosity on the movements of some invertebrate spermatozoa. 9. exp. Biol. 45, I13-39.

GrAY, J. (1955). The movement of sea-urchin spermatozoa. 9. exp. Biol. 32, 775-801.

Miles, C. A. \& Holwill, M. E. J. (1969). Asymmetric flagellar movement in relation to the orientation of the spore of Blastocladiella emersoni. F. exp. Biol. 50, 683-7.

Mirler, R. L. (1 $966 a$ ). Chemotaxis during fertilization in the hydroid Campanularia. J. exp. Zool. I6a, 23-44.

Miller, R. L. (1966b). Chemotaxis during fertilizacion in the hydroids Tubularia and Gonothyrea. Am. Zool. 6, 509.

NArtoh, Y. (I966). Reversal response elicited in non-beating cilia of Paramecium by membrane depolarization. Science, N.Y. 154, 660-2.

NAIton, Y. (1969). Control of the orientation of cilia by adenosinetriphosphate, calcium, and zinc in glycerol-extracted Paramecium caudatum. F. gen. Physiol. 53, 517-29.

\section{EXPLANATION OF PLATES}

\section{PLATE I}

Photographs of sperm paths near micropipettes filled with hydranth extract. The micropipette is near the lower edge or lower left corner of each photograph. The flash frequency was $9 \mathrm{~Hz}$. The arrows indicate the direction of sperm progreasion for paths which are referred to in the text. The direction is determined from the images of the sperm tails, which are visible in the original photographs and! a few of these copies.

\section{Ptate 2}

Figs. I -5 show the behaviour of the flagella of spermatozoa making chemotactic turns. Fig. 3 also illustrates the normal wave pattern. Fig. 6 is an example of aberrant wave patterns found with spermatozoa which are not fully activated. Fig. 7 is an example of the type of wave pattern found in spermatozoa after extended exposure to the chemotactant. These are negative prints from dark-field photomicrographs. 
Fournal of Experimental Biology, Vol. 52, No. 3

Plate I
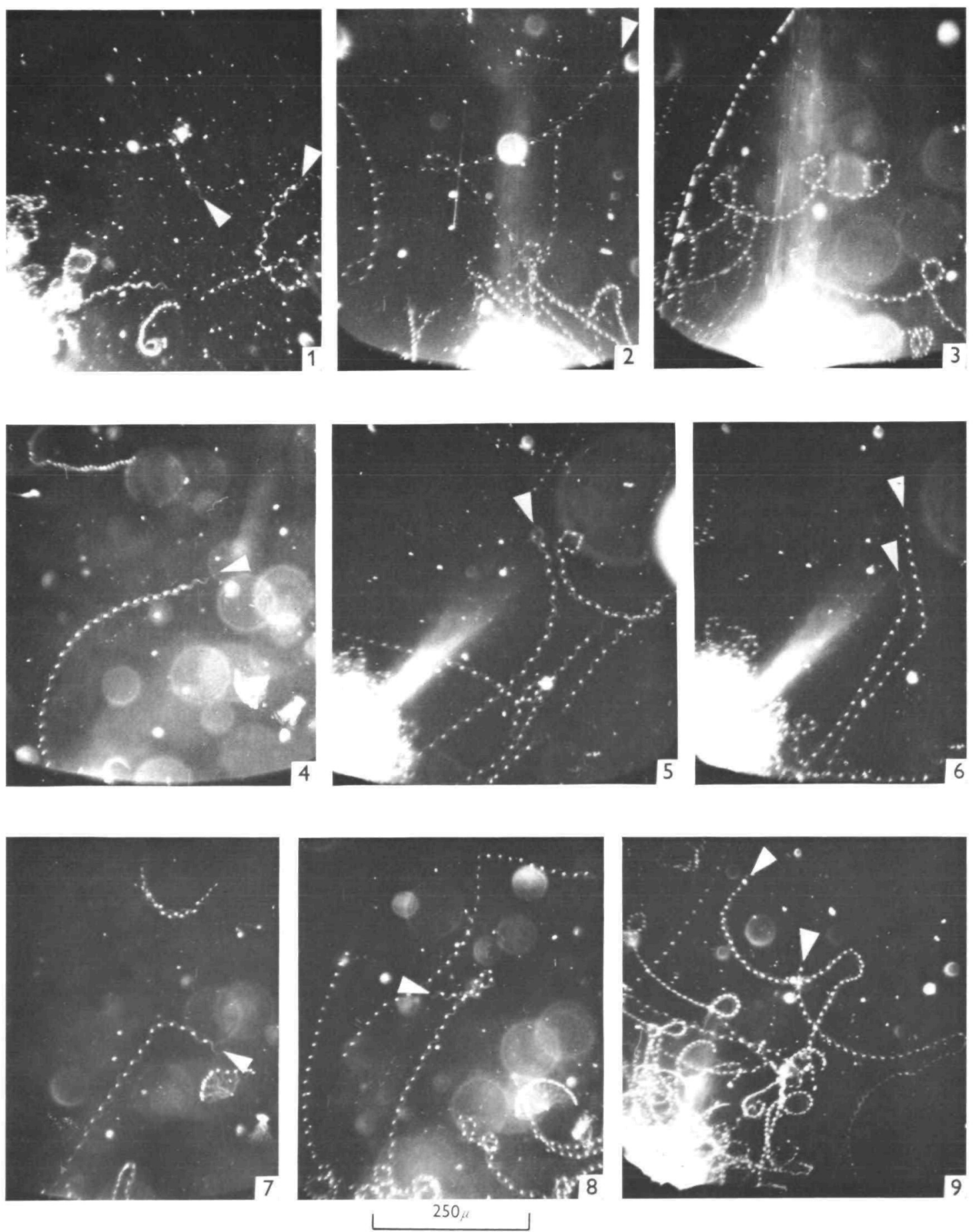

R. L. MILLER AND C. J. BROKAW

(Facing p. 706) 
Fournal of Experimental Biology, Vol. 52, No. 3

Plate 2
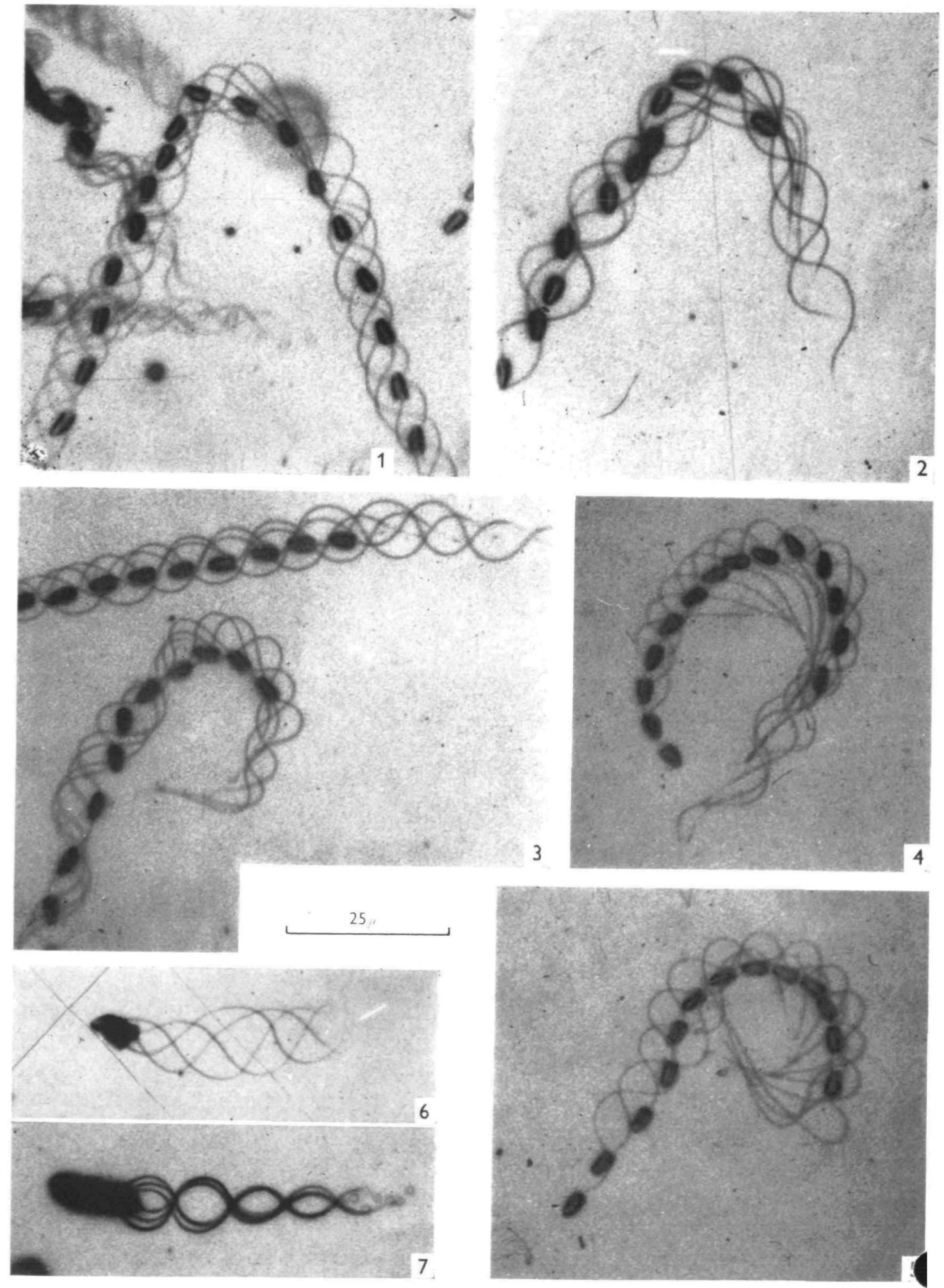

R. L. MILLER AND C. J. BROKAIV 